\title{
CALCULATION OF LATTICE RELAXATION IN SOME SUBSTITUTIONAL ALLOYS USING A GREEN FUNCTION
}

\author{
S.K. Das ${ }^{a}$ and S. Datta Roy (PaUl) ${ }^{b}$ \\ ${ }^{a}$ Azad Physics Centre, Department of Physics, Maulana Azad College \\ Calcutta 700 013, India \\ ${ }^{b}$ Department of Physics, Bethune College, Calcutta 700006, India
}

(Received July 1, 1998; revised version November 10, 1998;

in final form February 1, 1999)

In this paper we calculate nearest neighbour relaxation in some dilute substitutional alloys using a lattice static Green function and the Morse potential function. Distant neighbour relaxation is calculated by invoking a continuum approximation. The potential parameters for the unlike interactions are calculated using simple interpolation formulae. Using the above relaxation, we calculate volume changes in the above alloys. It is observed that the simple model predicts values which are in reasonably good agreement with the experimental values in all cases. The calculated values are also compared with those obtained from the Vegard law. For $\mathrm{Au}-\mathrm{Ag}$ alloy, our model reproduces both the correct sign and the magnitude of the volume change, whereas the Vegard law cannot even give the sign of the volume change. This shows the inherent strength of the present model.

PACS numbers: $61.72 . \mathrm{Bb}, 61.82 . \mathrm{Bg}$

\section{Introduction}

In studies of point defects, lattice relaxation plays an important role. Earlier, we have developed a consistent semi-discrete approach for the determination of the lattice relaxation and applied the same for the studies of substitutional defects in alkali halide solid solutions [1] and obtained good agreement with the experimental results. Besides the above method, there is another more powerful and theoretically more rigorous method based on a Green function to determine lattice relaxation. The Green function approach has been discussed in detail by Caldwell and Klein [2] and by Tewary [3]. In a previous paper [4] we have employed the Green function method to study the variation of nearest neighbour separation with concentration of defects in alkali halide solid solutions and have 
got encouraging results. In this paper, we try the above approach to estimate lattice relaxation and hence to calculate volume changes in some dilute metallic alloys. The method can also be applied to alloys with a finite concentration of defects with a suitable modification using Huang's idea [5]. Our work along this line on $\mathrm{K}$-Cs alloy is in progress and will be reported elsewhere. In addition to the simplicity and rigour, the present approach has the advantage that it minimises the labour of computation to a great extent.

In studies of point defects, it is usual to divide the crystal into two regions. Region I consists of the immediate neighbourhood of the point defect, and this region is treated in detail, atomistically. The remaining portion of the crystal is the region II. Using the continuum approximation for the region II, one puts the relaxation of an ion at a distance $r$ from the defect as $K / r^{2}$, where $K$ is a constant which we call the defect strength constant. For the region I, relaxation, $u_{\mathrm{nn}}$, of nearest neighbour to defect is written as $u_{\mathrm{nn}}=\xi r_{1}$, where $r_{1}$ is the nearest neighbour distance of the host crystal and $\xi$ is a parameter determined by the Green function discussed in the next section. Several different procedures for the determination of the constant $K$ exist in the literature [6-8]. Considering the simplicity, we follow the method suggested by Brauer [6]. The details of the procedure of determination of $K$ is also discussed in the next section. Utilising these relaxations and using the Morse potential function, we calculate volume changes in some low concentration binary alloys and compare them with the experimental values and those obtained from Vegard's law [9]. Considering the simplicity of the model, the agreement between the calculated and the experimental values seems to be quite appealing.

\section{Theory}

For a monoatomic crystal, the relaxation $\boldsymbol{U}\left({ }_{\alpha}^{l}\right)$ of an ion in the $l$-th cell along the $\alpha(=x, y, z)$ direction is given by

$$
\boldsymbol{U}\left(\begin{array}{l}
l \\
\alpha
\end{array}\right)=\sum_{l^{\prime}, \beta} G\left(\begin{array}{c}
l^{\prime} \\
\alpha \beta
\end{array}\right) \boldsymbol{F}\left(\begin{array}{l}
l \\
\beta
\end{array}\right)
$$

where the lattice static Green function $G\left({ }_{\alpha \beta}^{l i}\right)$ is defined by

$$
\begin{gathered}
G\left(\begin{array}{l}
l_{\alpha \beta}^{\prime} \\
l^{\prime}
\end{array}\right)=m \Phi^{-1}\left(l_{\alpha \beta}^{l^{\prime}}\right)=(1 / N) \sum_{q, j}\left[b(q, \alpha, j) b^{*}(q, \beta, j)\right. \\
\quad \times \exp \left[\mathrm{i} q \cdot\left(\boldsymbol{r}_{l}-\boldsymbol{r}_{l}^{\prime}\right)\right] / \omega^{2}(q, j),
\end{gathered}
$$

where $m$ is the mass of an ion of the pure crystal, $\Phi$ is a square $(3 N \times 3 N)$ force constant matrix of the pure crystal, $N$ is the number of cells in the crystal, $b(q, \alpha, j)$ are the eigenvectors and $\omega(q, j)$ are the eigenfrequencies and $j=1,2,3 . \boldsymbol{F}\left({ }_{\beta}^{l}\right)$ in Eq. (1) is the additional force experienced by an A (host) ion in the $l$-th cell along the $\beta(=x, y, z)$ direction due to the substitution by a foreign $\mathrm{B}$ (defect) ion at the origin. From Eqs. (1) and (2) we can find the displacement $\boldsymbol{U}\left({ }_{\alpha}^{l}\right)$ of an ion of the pure crystal provided the phonon spectrum of the perfect lattice is known. In the present paper, we have replaced $\omega^{2}(q, j)$ by an average Einstein frequency defined by

$$
\left\langle\omega^{2}\right\rangle=(1 / 3 N) \sum_{q, j} \omega^{2}(q, j)
$$


We have used the Morse potential for the purpose of calculation of $\left\langle\omega^{2}\right\rangle$ and $\boldsymbol{F}\left(l_{\beta}^{\prime}\right)$. For a two-body central potential $\phi\left(r_{i j}\right)$

$$
\begin{aligned}
\left\langle\omega^{2}\right\rangle & =[1 /(3 m)] \\
& \times \sum_{\alpha, l^{\prime}, \beta}\left[\left(r_{i j \alpha} r_{i j \beta} / r_{i j}^{2}\right) \phi^{\prime \prime}\left(r_{i j}\right)+\left(\delta_{\alpha \beta} / r_{i j}-r_{i j \alpha} r_{i j \beta} / r_{i j}^{3}\right) \phi^{\prime}\left(r_{i j}\right)\right] .
\end{aligned}
$$

For a fcc crystal and interaction up to the next nearest neighbour (nnn), we can write

$$
\left\langle\omega^{2}\right\rangle=(2 / m)\left[2 \phi_{1}^{\prime \prime}\left(r_{1}\right)+\frac{2 \sqrt{2}}{r_{0}} \phi_{1}^{\prime}\left(r_{1}\right)+\phi_{2}^{\prime \prime}\left(r_{2}\right)+\frac{\phi_{2}^{\prime}\left(r_{2}\right)}{r_{0}}\right],
$$

where $2 r_{0}$ is the lattice parameter, $r_{1}$ and $r_{2}$ are, respectively, the nearest neighbour (nn) and second nearest neighbour distances. Relaxation of the $\mathrm{A}$ ion at the $\mathrm{nn}$ site of the defect $\mathrm{B}$ ion at the origin is

$$
u\left(\begin{array}{l}
l=\mathrm{nn} \\
\alpha
\end{array}\right)=\frac{\boldsymbol{F}\left(\begin{array}{l}
l=\mathrm{nn} \\
\alpha
\end{array}\right)}{m\left\langle\omega^{2}\right\rangle}
$$

where the force $\boldsymbol{F}\left({ }_{\alpha}^{l}\right)$ is given by

$$
F=-\frac{\mathrm{d} \phi(r)}{\mathrm{d} r} \text { at } r=r_{1} \text {. }
$$

\subsection{Potential parameters for like and unlike atomic interactions}

The Morse potential function is usually written in the form

$$
\phi(r)=D\left[\exp \left(-2 \alpha\left(r-r_{0}\right)\right)-2 \exp \left(-\alpha\left(r-r_{0}\right)\right)\right],
$$

where $D, \alpha$ and $r_{0}$ are potential parameters. We shall, however, use a more convenient but equivalent form given by

$$
\phi(r)=\tilde{D}[\exp (-2 \tilde{\alpha}(r-\sigma))-\exp (-\tilde{\alpha}(r-\sigma))]
$$

where

$$
\tilde{D}=4 D, \quad \tilde{\alpha}=\alpha \quad \text { and } \quad \sigma=r_{0}-\frac{\ln 2}{\alpha} .
$$

The cohesive energy per particle is given by

$$
E=\frac{1}{2} \sum_{i j}^{\prime} \phi\left(r_{i j}\right)
$$

Considering interactions up to the second neighbour and the potential given by Eq. (8) or (9) we get

$$
E=\frac{n_{1}}{2} \phi_{1}\left(r_{1}\right)+\frac{n_{2}}{2} \phi_{2}\left(r_{2}\right)
$$

where $n_{1}$ and $n_{2}$ are the numbers of equivalent sites for the nearest neighbours (nn) and next nearest neighbours (nnn) and $r_{1}$ and $r_{2}$ are the respective separations. $n_{1}=12, n_{2}=6$ and $r_{2}=\sqrt{2} r_{1}$ for a fcc crystal. We now have the equilibrium condition

$$
\left[\frac{\partial E}{\partial r_{1}}\right]_{r_{1}=\tilde{r}_{1}}=0
$$


where $\tilde{r}_{1}$ is the harmonic value of the nearest neighbour separation. The expression for the harmonic bulk modulus can be written as

$$
\chi=V \frac{\mathrm{d}^{2} E}{\mathrm{~d} V^{2}}
$$

where $V$ is the volume per atom. $V=r_{1}^{3} / \sqrt{2}$ for a fcc crystal. Equations (12), (13) and (14) can be used to determine the potential parameters for pure metals, using the experimental values of the cohesive energy corrected for zero point effect, the harmonic values of the lattice parameter, and bulk modulus. These parameters along with the data used for their evaluation are listed in Table I. The potential

TABLE I

Parameters of the Morse potential for pure metals and data used for their evaluation. The harmonic lattice parameter is denoted by $d . d$ is calculated by using harmonic density $\rho$ and atomic weight. Cohesive energy is denoted by $E$. The harmonic bulk modulus is denoted by $\chi$.

\begin{tabular}{c|r|c|c|c|c|c|c}
\hline \hline Metal & $\begin{array}{c}\rho[12] \\
{\left[\mathrm{g} \mathrm{cm}^{-3}\right]}\end{array}$ & $\begin{array}{c}d \\
{\left[10^{-8} \mathrm{~cm}\right]}\end{array}$ & $\begin{array}{c}E[13] \\
{\left[10^{-12}\right.} \\
\left.\frac{\mathrm{erg}}{\mathrm{atom}}\right]\end{array}$ & $\begin{array}{c}\chi[12] \\
{\left[10^{12}\right.} \\
\left.\frac{\text { dyne }}{\mathrm{cm}^{2}}\right]\end{array}$ & $\begin{array}{c}D \\
{\left[10^{-13} \mathrm{erg}\right]}\end{array}$ & $\begin{array}{c}\alpha \\
{\left[10^{8} \mathrm{~cm}\right]}\end{array}$ & $\begin{array}{c}r_{0} \\
{\left[10^{-8} \mathrm{~cm}\right]}\end{array}$ \\
\hline $\mathrm{Au}$ & 19.551 & 4.0600 & 6.0552 & 1.814 & 8.9181 & 1.6300 & 2.9214 \\
$\mathrm{Cu}$ & 9.083 & 3.5950 & 5.6067 & 1.433 & 7.7988 & 1.3980 & 2.6230 \\
$\mathrm{Ni}$ & 9.020 & 3.5096 & 7.1044 & 1.888 & 9.8550 & 1.4100 & 2.5629 \\
$\mathrm{Ag}$ & 10.661 & 4.0657 & 4.7416 & 1.099 & 6.8170 & 1.4250 & 2.9439
\end{tabular}

parameters for unlike interactions have been determined following the combination rules adopted for unlike molecular interactions in gas pairs on the basis of the Morse potential function [10]. The combination rules for unlike interactions may be summarized in the following way. If the potential parameters for A-type atoms are $D_{\mathrm{A}}, \alpha_{\mathrm{A}}$ and $\sigma_{\mathrm{A}}$, and $D_{\mathrm{B}}, \alpha_{\mathrm{B}}$ and $\sigma_{\mathrm{B}}$ are the corresponding parameters for the $\mathrm{B}$-type atoms, then the parameters for the $\mathrm{AB}$ interaction are determined from the interpolation formulae [10],

$$
\begin{aligned}
& D_{\mathrm{AB}}=\sqrt{D_{\mathrm{A}} D_{\mathrm{B}}}, \\
& \alpha_{\mathrm{AB}}=0.5\left(\alpha_{\mathrm{A}}+\alpha_{\mathrm{B}}\right), \\
& \sigma_{\mathrm{AB}}=\sqrt{\sigma_{\mathrm{A}} \sigma_{\mathrm{B}}} .
\end{aligned}
$$

The parameter $r_{\mathrm{OAB}}$ is related to the parameter $\sigma_{\mathrm{AB}}$ by

$$
r_{0 \mathrm{AB}}=\sigma_{\mathrm{AB}}+\frac{\ln 2}{\alpha_{\mathrm{AB}}} .
$$

The parameters determined in this manner are listed in Table II. 
TABLE II

Parameters of the Morse potential for AB interactions from combination rules.

\begin{tabular}{l|c|c|c}
\hline $\begin{array}{l}\text { Alloy } \\
(\mathrm{A}-\mathrm{B})\end{array}$ & $\begin{array}{c}D \\
{\left[10^{-13} \mathrm{erg}\right]}\end{array}$ & $\begin{array}{c}\alpha \\
{\left[10^{8} \mathrm{~cm}\right]}\end{array}$ & $\begin{array}{c}r_{0} \\
{\left[10^{-8} \mathrm{~cm}\right]}\end{array}$ \\
\hline $\mathrm{Au}-\mathrm{Cu}$ & 8.3397 & 1.5140 & 2.7621 \\
$\mathrm{Cu}-\mathrm{Ni}$ & 8.7832 & 1.4050 & 2.5941 \\
$\mathrm{Cu}-\mathrm{Ag}$ & 7.2914 & 1.4115 & 2.7775 \\
$\mathrm{Au}-\mathrm{Ag}$ & 7.7971 & 1.5275 & 2.9306
\end{tabular}

\subsection{Volume change}

The expression for the volume change can be obtained from Eshelby's continuum theory of elasticity [11] and is given by (for fcc crystal)

$$
\frac{1}{C} \frac{\Delta V}{V}=4 \sqrt{2} \pi \xi
$$

where $C$ is the fractional concentration of the impurity ion. The parameter $\xi$ determines the nearest neighbour relaxation and is determined by the equation

$$
u(110)=u_{\mathrm{nn}}=\xi r_{1},
$$

where the merging parameter $k$ is taken to be unity following Brauer's [6] assumption. Hence Eq. (19) reduces to.

$$
\frac{1}{C} \frac{\Delta V}{V}=4 \sqrt{2} \pi \xi
$$

Considering the image term [11], the above expressions for the volume changes will be multiplied by $\frac{3(1-\nu)}{1+\nu}$, where $\nu$ is the Poisson ratio. For almost all metals, $\nu=1 / 3$. Hence, we get volume change for fcc crystals,

$$
\frac{1}{C} \frac{\Delta V}{V}=6 \sqrt{2} \pi \xi
$$

\section{Results and discussion}

From Table III, we note that, except $\mathrm{Cu}-\mathrm{Au}$ alloy, the calculated values agree with the experimental values within reasonable limits (within 30\%). Signs of volume changes come out correctly in all cases. Considering the very large uncertainty in the experimental measurement this agreement is not bad. The discrepancy in $\mathrm{Cu}-\mathrm{Au}$ alloy seems to be due to the fact that the Brauer approximation $(k=1)$ overestimates the distant neighbour relaxation in this case. Another interesting point may be noted in connection with $\mathrm{Au}-\mathrm{Ag}$ alloy. Here the Vegard law cannot predict even the sign of the experimental volume change, whereas the present model reproduces both the sign and the magnitude. This shows the inherent strength of the present model. 
TABLE III

Evaluated volume changes for several alloys and comparison with experimental results.

\begin{tabular}{c|c|c|c|c}
\hline \hline $\begin{array}{c}\text { Alloy } \\
(\mathrm{A}-\mathrm{B})\end{array}$ & $\xi$ & $\begin{array}{c}10^{2} \frac{1}{C} \frac{\Delta V}{V} \\
\text { (Calculated) }\end{array}$ & $\begin{array}{c}10^{2} \frac{1}{C} \frac{\Delta V}{V} \\
\text { (Vegard's law) }\end{array}$ & $\begin{array}{c}10^{2} \frac{1}{C} \frac{\Delta V}{V} \\
\text { (Experimental) }\end{array}$ \\
\hline $\mathrm{Au}-\mathrm{Cu}$ & -0.0091 & -24.26 & -30.57 & $-28^{a},-26.87^{b}$ \\
$\mathrm{Cu}-\mathrm{Au}$ & 0.0276 & 73.57 & 44.04 & $48^{a}, 42.66^{b}$ \\
$\mathrm{Cu}-\mathrm{Ni}$ & -0.0024 & -6.40 & -6.96 & $-8.5^{a},-9.00^{b}$ \\
$\mathrm{Ni}-\mathrm{Cu}$ & 0.0021 & 5.70 & 7.48 & $7^{a}, 7.00^{b}$ \\
$\mathrm{Cu}-\mathrm{Ag}$ & 0.0210 & 55.99 & 44.65 & $44^{a}, 61.27^{b}$ \\
$\mathrm{Ag}-\mathrm{Cu}$ & -0.0109 & -29.05 & -30.87 & $-28^{a},-26.41^{b}$ \\
$\mathrm{Au}-\mathrm{Ag}$ & -0.0005 & -1.33 & 0.42 & $-0.6^{a},-1.27^{c}$ \\
\hline
\end{tabular}

${ }^{a}$ King [14]; ${ }^{b}$ Pearson [15]; ${ }^{c}$ Barrett [16].

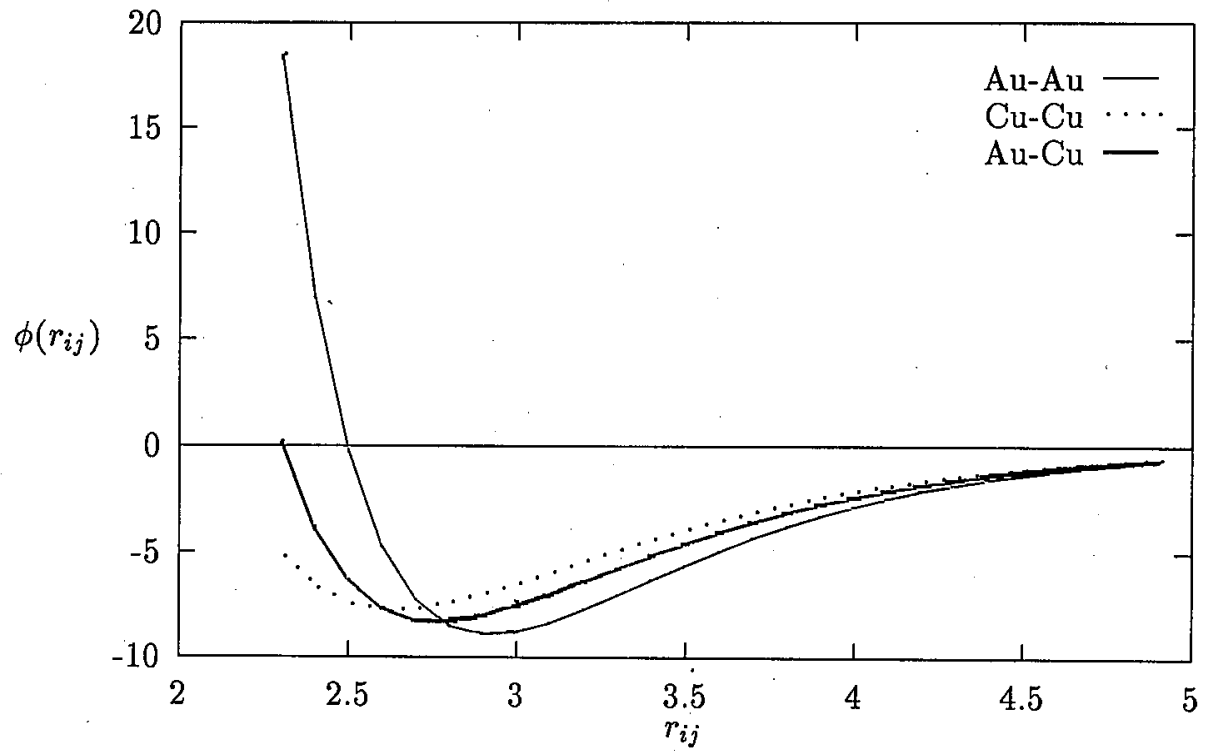

Fig 1. Comparison plots of $\mathrm{Au}-\mathrm{Au}, \mathrm{Cu}-\mathrm{Cu}$ and $\mathrm{Au}-\mathrm{Cu}$ interaction potentials. $r_{i j}$ is in $10^{-8} \mathrm{~cm}$ and $\phi\left(r_{i j}\right)$ is in $10^{-13} \mathrm{erg}$.

In order to test the quality of the interpolation (suggested by Saran [10]) construction of the $\mathrm{A}-\mathrm{B}$ interaction potential, using the $\mathrm{A}-\mathrm{A}$ and $\mathrm{B}-\mathrm{B}$ potential parameters, we have recalculated the volume changes for all the alloys studied using an alternative choice, $D_{\mathrm{AB}}=\frac{1}{2}\left(D_{\mathrm{A}}+D_{\mathrm{B}}\right), \alpha_{\mathrm{AB}}=\sqrt{\alpha_{\mathrm{A}} \alpha_{\mathrm{B}}}$ and $\sigma_{\mathrm{AB}}=\frac{1}{2}\left(\sigma_{\mathrm{A}}+\sigma_{\mathrm{B}}\right)$, to determine the potential parameters for the $A-B$ interaction. The recalculated results do not show any major change in the values. For three alloys $(\mathrm{Au}-\mathrm{Cu}$, 


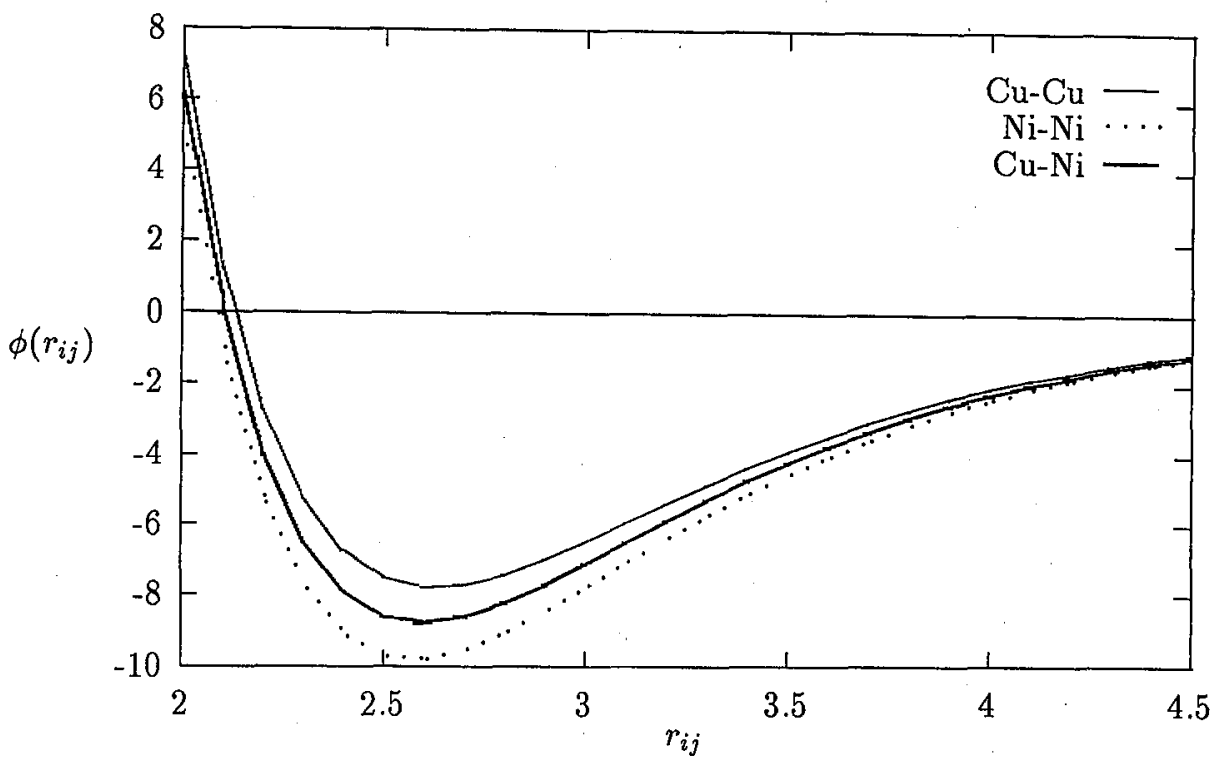

Fig 2. Comparison plots of $\mathrm{Cu}-\mathrm{Cu}, \mathrm{Ni}-\mathrm{Ni}$ and $\mathrm{Cu}-\mathrm{Ni}$ interaction potentials. $r_{i j}$ is in $10^{-8} \mathrm{~cm}$ and $\phi\left(r_{i j}\right)$ is in $10^{-13} \mathrm{erg}$.

$\mathrm{Cu}-\mathrm{Au}$ and $\mathrm{Ni}-\mathrm{Cu}$ ) disagreement between theory and experiment increases by about 3 to 9 per cent, whereas for the rest four alloys recalculated values show better agreement (by about 5 to 8 per cent) with the experimental values. This shows that the choice of Saran [10] is not unique, and other alternative choices which do not alter the calculated results appreciably exist. We get further justification in favour of the above statement from the fact that the nature of the potential curves does not alter significantly for the alloys by using the above alternative choice. In order to show the quality of the potential construction we have plotted the potentials for pure metals and their alloys in the same graph (Figs. 1 and 2) for the alloys $\mathrm{Au}-\mathrm{Cu}$ and $\mathrm{Cu}-\mathrm{Ni}$ respectively.

\section{Acknowledgment}

We are grateful to the anonymous referee for constructive suggestions and a useful reference for guidance. We are also grateful to Prof. (Emeritus) S. Sengupta, Department of Physics, Jadavpur University, Calcutta, for fruitful discussion. Finally one of us (S.K.D.) is thankful to Prof. T. Dey, Head of the Department of Physics, Maulana Azad College, Calcutta, for encouragement during the investigation of the problem and to Prof. S. Datta, Head of the Department of Physics, Presidency College, Calcutta, for allowing him to use one of the departmental computers. 


\section{References}

[1] S. Paul, S. Sengupta, Phys. Status Solidi B 68, 703 (1975).

[2] R.F. Caldwell, M.V. Klein, Phys. Rev. 158, 851 (1967).

[3] V.K. Tewary, Adv. Phys. 22, 757 (1973).

[4] S. Datta Roy (Paul), S. Sengupta, Phys. Status Solidi B 162, 89 (1990).

[5] K. Huang, Proc. R. Soc. Lond. A 190, 102 (1947).

[6] P. Brauer, Z. Naturforsch. 7a, 372 (1952).

[7] B.G. Dick, T.P. Das, Phys. Rev. 127, 1053 (1962).

[8] J.R. Hardy, J. Phys. Chem. Solids 29, 2009 (1968).

[9] L. Vegard, Z. Phys. 5, 17 (1921).

[10] A. Saran, Ind. J. Phys. 37, 491 (1963).

[11] J.D. Eshelby, Solid State Physics, Eds. F. Seitz, D. Turnbull, Vol. 3, Academic Press, New York 1956.

[12] G. Simmons, H. Wang, Temperature Variations of Elastic Constants and Calculated Aggregate Properties, MIT Press, Cambridge, Mass. 1971.

[13] C. Kittel, Introduction to Solid State Physics, Wiley, London 1968, p. 78, 180.

[14] H.W. King, J. Mater. Sci. 1, 79 (1965).

[15] W.B. Pearson, A Handbook of Lattice Spacings and Structures of Metals and Alloys, Pergamon, London 1958.

[16] C.S. Barrett, Structure of Metals, 2nd ed., McGraw-Hill, New York 1952, p. 222. 